\title{
First-order Asymptotics of the BICM Mutual Information: Uniform vs. Nonuniform Distributions
}

\author{
Erik Agrell \\ Department of Signals and Systems \\ Chalmers University of Technology \\ SE-41296 Göteborg \\ Sweden \\ Email: agrell@chalmers.se
}

\author{
Alex Alvarado \\ Department of Engineering \\ University of Cambridge \\ Cambridge CB2 1PZ \\ United Kingdom \\ Email: alex.alvarado@ieee.org
}

\begin{abstract}
A linear, invertible transform is defined between two vectors or matrices as a tool for analyzing the bit-interleaved coded modulation (BICM) mutual information in the wideband regime. The transform coefficients depend on a set of real values, which can be interpreted as probabilities. The transform relates any BICM system with a nonuniform input distribution to another BICM system with a uniform distribution. Numerical evidence suggests that the two systems have the same first-order behavior, which would make possible to analyze nonuniform BICM systems based on known properties of uniform BICM systems.
\end{abstract}

\section{INTRODUCTION}

In 1992, Zehavi introduced the so-called bit-interleaved coded modulation (BICM) [1], and it has since then been rapidly adopted in commercial systems such as wireless and wired broadband access networks, 3G/4G telephony, and digital video broadcasting [2]. An achievable rate for BICM systems is the so-called BICM mutual information (BICMMI) defined as the sum of the mutual informations for each bit separately [3] [4]. The asymptotic behavior of the BICM$\mathrm{MI}$ at low signal-to-noise ratio (SNR), i.e., in the wideband regime, was studied in [5]-[9] as a function of the alphabet and the binary labeling, assuming a uniform input distribution.

Probabilistic shaping for BICM, i.e., varying the probabilities of the bit streams, was first proposed in [10], [11] and developed further in [12]-[14]. Probabilistic shaping offers another degree of freedom in the BICM design, which can be used to make the discrete input distribution more similar to a Gaussian distribution. This is particularly advantageous at low and medium SNR.

In this paper, the first-order asymptotic behavior of the BICM-MI is analyzed for BICM systems with nonuniform distributions. A linear transform is introduced, which establishes an equivalence between an arbitrary nonuniform constellation and another constellation with uniform probabilities, in the sense that the BICM-MI of the two constellations have the same first-order asymptote. Since the uniform case has been investigated in detail and is now well understood (see references above, in particular [9]), the new transform offers an instrument to generalize such results into the nonuniform case.

Notation: Matrices are denoted by block letters $\mathbb{A}$ and vectors are row vectors. The Euclidean norm of $\boldsymbol{a}$ is denoted by $\|\boldsymbol{a}\|$. Random variables are denoted by capital letters $A$ and random vectors by boldface capital vectors $\boldsymbol{A}$. The binary set is denoted by $\mathcal{B} \triangleq\{0,1\}$ and the negation of a bit $b$ is denoted by $\bar{b}=1-b$. Expectations are denoted by $\mathbb{E}$.

\section{System ModeL}

We consider the generic BICM scheme in Fig. 1. The transmitter is, in the simplest case, a single binary encoder concatenated with an interleaver and a memoryless mapper $\Phi$. Multiple encoders and/or interleavers may be needed to achieve probabilistic shaping [11]-[14]. The mapper $\Phi$ is defined via the alphabet $\mathbb{X} \triangleq\left[\boldsymbol{x}_{0}^{\mathrm{T}}, \ldots, \boldsymbol{x}_{M-1}^{\mathrm{T}}\right]^{\mathrm{T}}$, where $M=$ $2^{m}$ and $\boldsymbol{x}_{i} \in \mathbb{R}^{N}$ for $i=0, \ldots, M-1$. Each symbol $\boldsymbol{x}_{i}$ is labeled by the $m$ bits that form the base- 2 representation $\left[n_{i, 0}, n_{i, 1}, \ldots, n_{i, m-1}\right]$ of the integer $i$. As a consequence of this enumeration convention, the constellation $\mathbb{X}$ is labeled by the natural binary code. However, this is merely a notational convention, which does not limit the applicability of the results. An arbitrary binary labeling for the same alphabet can be analyzed simply by reordering the rows of $\mathbb{X}$.

Assuming independent, but possibly nonuniformly distributed, bits $C_{0}, \ldots, C_{m-1}$ at the input of the mapper (cf. Fig. 1), the probability that the symbol $\boldsymbol{x}_{i}$ will be transmitted is [9, eq. (30)] [14, eq. (8)] [15, eq. (9)]

$$
P_{i}=\prod_{k=0}^{m-1} P_{C_{k}}\left(n_{i, k}\right)
$$

for $i=0, \ldots, M-1$, where $P_{C_{k}}(u)$ for $u \in \mathcal{B}$ is the probability of $C_{k}=u$. Since $P_{C_{k}}(1)=1-P_{C_{k}}(0)$, the distribution $\mathbb{P}$ is fully specified by the set of bit probabilities $\left[P_{C_{0}}(0), \ldots, P_{C_{m-1}}(0)\right]$. A constellation is defined as the pair $[\mathbb{X}, \mathbb{P}]$, where $\mathbb{P} \triangleq\left[P_{0}, \ldots, P_{M-1}\right]^{\mathrm{T}}$ is the input distribution. An important special case is the uniform distribution, for which $P_{C_{k}}(0)=1 / 2$ for $k=0, \ldots, m-1$ and $\mathbb{P}=\mathbb{U}_{m} \triangleq$ $[1 / M, \ldots, 1 / M]^{\mathrm{T}}$.

Throughout this paper, we assume that $0<P_{C_{k}}(0)<1$ for all $k=0, \ldots, m-1$, i.e., we assume that all constellation points are used with a nonzero probability. If that was not the case, the cardinality of the constellation should be reduced. Hence $P_{i}>0$ for $i=0, \ldots, M-1$. 


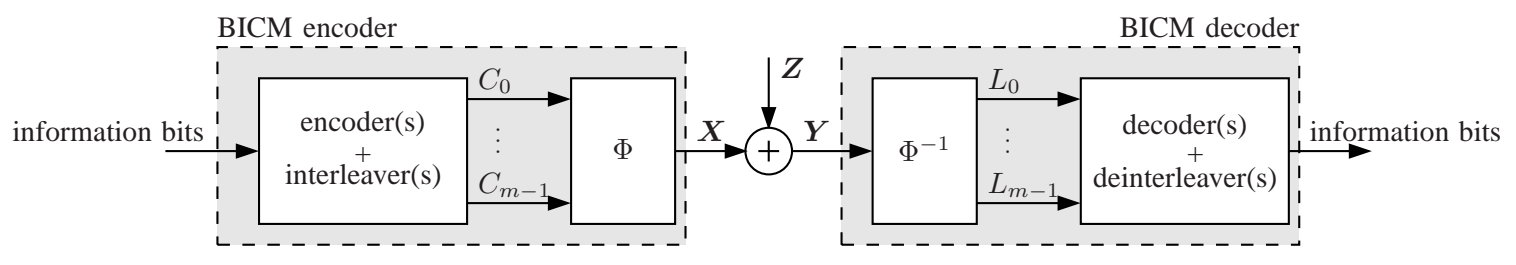

Fig. 1. A generic BICM system, consisting of a transmitter, an AWGN channel, and a receiver.

We consider transmissions over a discrete-time memoryless additive white Gaussian noise channel. The received vector at any discrete time instant is

$$
\boldsymbol{Y}=\boldsymbol{X}+\boldsymbol{Z}
$$

where $\boldsymbol{X}$ is the channel input and $\boldsymbol{Z}$ is a Gaussian noise with zero mean and variance $N_{0} / 2$ in each dimension. The SNR is defined as

$$
S N R \triangleq \frac{E_{\mathrm{s}}}{N_{0}}=R_{\mathrm{c}} \frac{E_{\mathrm{b}}}{N_{0}},
$$

where $R_{\mathrm{c}}$ is the transmission rate in information bits per symbol, $E_{\mathrm{s}} \triangleq \mathbb{E}\left[\|\boldsymbol{X}\|^{2}\right]$ is the average symbol energy, and $E_{\mathrm{b}} \triangleq E_{\mathrm{s}} / R_{\mathrm{c}}$ is the average bit energy.

At the receiver, using the channel output $\boldsymbol{Y}$, the demapper $\Phi^{-1}$ computes metrics $L_{k}$ for the individual coded bits $C_{k}$ with $k=0, \ldots, m-1$, usually in the form of logarithmic likelihood ratios. These metrics are then passed to the deinterleaver(s) and decoder(s) to obtain an estimate of the information bits.

\section{MUTUAL INFORMATION}

The mutual information (MI) in bits per channel use between the random vectors $\boldsymbol{X}$ and $\boldsymbol{Y}$ is

$$
I_{\boldsymbol{X}}(\boldsymbol{X} ; \boldsymbol{Y}) \triangleq \mathbb{E}\left[\log _{2} \frac{p_{\boldsymbol{Y} \mid \boldsymbol{X}}(\boldsymbol{Y} \mid \boldsymbol{X})}{p_{\boldsymbol{Y}}(\boldsymbol{Y})}\right]
$$

where the expectation is taken over the joint pdf $p_{\boldsymbol{X}, \boldsymbol{Y}}(\boldsymbol{x}, \boldsymbol{y})$ and the conditional transition pdf is

$$
p_{\boldsymbol{Y} \mid \boldsymbol{X}}(\boldsymbol{y} \mid \boldsymbol{x})=\frac{1}{\left(N_{0} \pi\right)^{N / 2}} \exp \left(-\frac{\|\boldsymbol{y}-\boldsymbol{x}\|^{2}}{N_{0}}\right) .
$$

The conditional MI is defined as the MI between $\boldsymbol{X}$ and $\boldsymbol{Y}$ conditioned on the value of the $k$ th bit at the input of the mapper, i.e.,

$$
I_{\boldsymbol{X} \mid C_{k}=u}(\boldsymbol{X} ; \boldsymbol{Y}) \triangleq \mathbb{E}\left[\log _{2} \frac{p_{\boldsymbol{Y} \mid \boldsymbol{X}, C_{k}}(\boldsymbol{Y} \mid \boldsymbol{X}, u)}{p_{\boldsymbol{Y}, C_{k}}(\boldsymbol{Y} \mid u)}\right],
$$

where the expectation is taken over the conditional joint pdf $p_{\boldsymbol{X}, \boldsymbol{Y} \mid C_{k}}(\boldsymbol{x}, \boldsymbol{y} \mid u)$.

We are interested in the BICM-MI, defined as [3]-[5], [8]

$$
I(S N R) \triangleq m I_{\boldsymbol{X}}(\boldsymbol{X} ; \boldsymbol{Y})-\sum_{k=0}^{m-1} \sum_{u \in \mathcal{B}} P_{C_{k}}(u) I_{\boldsymbol{X} \mid C_{k}=u}(\boldsymbol{X} ; \boldsymbol{Y})
$$

We will analyze the right-hand side of (6) as a function of $S N R$, which means either varying $N_{0}$ for fixed constellation or, equivalently, rescaling the alphabet $\mathbb{X}$ linearly for fixed input distribution and $N_{0}$.

Martinez et al. [3] recognized the BICM decoder in Fig. 1 as a mismatched decoder and showed that the BICM-MI in (6) corresponds to an achievable rate of such a decoder. This means that reliable transmission using a BICM system at rate $R_{\mathrm{c}}$ is possible if and only if $R_{\mathrm{c}} \leq I(S N R)$. Since from (3) $S N R / R_{\mathrm{c}}=E_{\mathrm{b}} / N_{0}$, the inequality $R_{\mathrm{c}} \leq I(S N R)$ gives

$$
\frac{E_{\mathrm{b}}}{N_{0}} \geq \frac{S N R}{I(S N R)}
$$

for any $S N R$. Specifically, for asymptotically low SNR (or equivalently asymptotically low rates $R_{\mathrm{c}}$, which means in the wideband regime), the average bit energy needed for reliable transmission is lower-bounded by $E_{\mathrm{b}} / N_{0} \geq \alpha^{-1}$, where

$$
\alpha \triangleq \lim _{S N R \rightarrow 0^{+}} \frac{I(S N R)}{S N R} .
$$

Furthermore, $\alpha^{-1} \geq \log _{\mathrm{e}} 2=-1.59 \mathrm{~dB}$, since no communication system can surpass the Shannon limit (SL).

\section{A Distribution-DEPENDENT TRANSFORM}

In this section, we define a linear transform between vectors or matrices, which depends on the input distribution $\mathbb{P}$ via the bit probabilities $\left[P_{C_{0}}(0), \ldots, P_{C_{m-1}}(0)\right]$.

For all $i=0, \ldots, M-1$ and $j=0, \ldots, M-1$, we define the transform coefficients

$\gamma_{i, j} \triangleq \prod_{k=0}^{m-1}\left[(-1)^{\bar{n}_{i, k} n_{j, k}} \sqrt{P_{C_{k}}(0)}+(-1)^{n_{i, k} \bar{n}_{j, k}} \sqrt{P_{C_{k}}(1)}\right]$.

Given probabilities $\left[P_{C_{0}}(0), \ldots, P_{C_{m-1}}(0)\right]$, the transform $\stackrel{\mathbb{X}}{\mathbb{X}}=\left[\stackrel{\circ}{x}_{0}^{\mathrm{T}}, \ldots, \stackrel{x}{\boldsymbol{x}}_{M-1}^{\mathrm{T}}\right]^{\mathrm{T}}$ of a matrix (or vector) $\mathbb{X}=$ $\left[\boldsymbol{x}_{0}^{\mathrm{T}}, \ldots, \boldsymbol{x}_{M-1}^{\mathrm{T}}\right]^{\mathrm{T}}$ with $M=2^{m}$ rows is now defined as

$$
\stackrel{\circ}{\boldsymbol{x}}_{i} \triangleq \sum_{j=0}^{M-1} \boldsymbol{x}_{j} \gamma_{i, j} \sqrt{P_{j}}, \quad i=0, \ldots, M-1,
$$

with $P_{j}$ given by (1). For equally likely symbols, i.e., $\mathbb{P}=\mathbb{U}_{m}$, the new transform becomes the identity operation $\mathbb{X}=\mathbb{X}$, because then $\gamma_{i, i}=\sqrt{M}$ for $i=1, \ldots, M$ and $\gamma_{i, j}=0$ for $i \neq j$.

Theorem 1: Given probabilities $\left[P_{C_{0}}(0), \ldots, P_{C_{m-1}}(0)\right]$, the inverse transform $\mathbb{X}=\left[\boldsymbol{x}_{0}^{\mathrm{T}}, \ldots, \boldsymbol{x}_{M-1}^{\mathrm{T}}\right]^{\mathrm{T}}$ of a matrix (or vector) $\stackrel{\circ}{\mathbb{X}}=\left[\stackrel{\circ}{\boldsymbol{x}}_{0}^{\mathrm{T}}, \ldots, \stackrel{\leftrightarrow}{\boldsymbol{x}}_{M-1}^{\mathrm{T}}\right]^{\mathrm{T}}$ is

$$
\boldsymbol{x}_{j}=\frac{1}{M \sqrt{P_{j}}} \sum_{i=0}^{M-1}{\stackrel{\circ}{\boldsymbol{x}_{i}}}_{i, j}, \quad j=0, \ldots, M-1 .
$$


Before proving Theorem 1, we need to establish a lemma.

Lemma 1: Let $f_{k, u}$ for $k=0, \ldots, m-1$ and $u \in \mathcal{B}$ be any real numbers. Then

$$
\sum_{i=0}^{M-1} \prod_{k=0}^{m-1} f_{k, n_{i, k}}=\prod_{k=0}^{m-1}\left(f_{k, 0}+f_{k, 1}\right) .
$$

Proof: A summation over $i=0, \ldots, 2^{m}-1$ is equivalent to $m$ sums over $i_{k} \in \mathcal{B}$, where $k=0, \ldots, m-1$ and $i=$ $i_{0}+2 i_{1}+\cdots+2^{m-1} i_{m-1}$. With this notation, $n_{i, k}=i_{k}$ and

$$
\begin{aligned}
\sum_{i=0}^{M-1} \prod_{k=0}^{m-1} f_{k, n_{i, k}} & =\sum_{i_{0} \in \mathcal{B}} \sum_{i_{1} \in \mathcal{B}} \cdots \sum_{i_{m-1} \in \mathcal{B}} \prod_{k=0}^{m-1} f_{k, i_{k}} \\
& =\sum_{i_{0} \in \mathcal{B}} \sum_{i_{1} \in \mathcal{B}} \cdots \sum_{i_{m-1} \in \mathcal{B}} f_{0, i_{0}} f_{1, i_{1}} \cdots f_{m-1, i_{m-1}} \\
& =\sum_{i_{0} \in \mathcal{B}} f_{0, i_{0}} \sum_{i_{1} \in \mathcal{B}} f_{1, i_{1}} \cdots \sum_{i_{m-1} \in \mathcal{B}} f_{m-1, i_{m-1}} \\
& =\prod_{k=0}^{m-1} \sum_{i_{k} \in \mathcal{B}} f_{k, i_{k}} .
\end{aligned}
$$

This Lemma will now be used in the proof of Theorem 1.

Proof of Theorem 1:

For $j=0, \ldots, M-1$,

$$
\begin{aligned}
\sum_{i=0}^{M-1} \stackrel{\circ}{x}_{i} \gamma_{i, j} & =\sum_{i=0}^{M-1} \gamma_{i, j} \sum_{l=0}^{M-1} \boldsymbol{x}_{l} \gamma_{i, l} \sqrt{P_{l}} \\
& =\sum_{l=0}^{M-1} \boldsymbol{x}_{l} \sqrt{P_{l}} \sum_{i=0}^{M-1} \gamma_{i, l} \gamma_{i, j}
\end{aligned}
$$

The inner sum can be expanded using the definition of $\gamma_{i, j}$ in (7) as

$$
\begin{aligned}
& \sum_{i=0}^{M-1} \gamma_{i, l} \gamma_{i, j}=\sum_{i=0}^{M-1} \prod_{k=0}^{m-1}\left[(-1)^{\bar{n}_{i, k} n_{l, k}} \sqrt{P_{C_{k}}(0)}\right. \\
& \left.\quad+(-1)^{n_{i, k} \bar{n}_{l, k}} \sqrt{P_{C_{k}}(1)}\right] \\
& \quad \cdot\left[(-1)^{\bar{n}_{i, k} n_{j, k}} \sqrt{P_{C_{k}}(0)}\right. \\
& \left.\quad+(-1)^{n_{i, k} \bar{n}_{j, k}} \sqrt{P_{C_{k}}(1)}\right] \\
& =\sum_{i=0}^{M-1} \prod_{k=0}^{m-1}\left[(-1)^{\bar{n}_{i, k}\left(n_{l, k}+n_{j, k}\right)} P_{C_{k}}(0)\right. \\
& \quad+(-1)^{\bar{n}_{i, k} n_{l, k}+n_{i, k} \bar{n}_{j, k} \sqrt{P_{C_{k}}(0) P_{C_{k}}(1)}} \\
& \quad+(-1)^{n_{i, k} \bar{n}_{l, k}+\bar{n}_{i, k} n_{j, k} \sqrt{P_{C_{k}}(0) P_{C_{k}}(1)}} \\
& \left.\quad+(-1)^{n_{i, k}\left(\bar{n}_{l, k}+\bar{n}_{j, k}\right)} P_{C_{k}}(1)\right] \\
& =\sum_{i=0}^{M-1} \prod_{k=0}^{m-1}(-1)^{n_{i, k}\left(n_{l, k}+n_{j, k}\right)} \\
& \quad \cdot\left[(-1)^{n_{l, k}+n_{j, k}} P_{C_{k}}(0)+P_{C_{k}}(1)\right. \\
& \left.\quad+(-1)^{n_{i, k}}\left[(-1)^{n_{l, k}}+(-1)^{n_{j, k}}\right] \sqrt{P_{C_{k}}(0) P_{C_{k}}(1)}\right],
\end{aligned}
$$

where the last equality follows by repeatedly using the identities $\bar{u}=1-u$ and $(-1)^{u}=(-1)^{-u}$ for $u \in \mathcal{B}$. We apply Lemma 1 with

$$
\begin{aligned}
& f_{k, u}=(-1)^{u\left(n_{l, k}+n_{j, k}\right)}\left[(-1)^{n_{l, k}+n_{j, k}} P_{C_{k}}(0)+P_{C_{k}}(1)\right. \\
&\left.+(-1)^{u}\left[(-1)^{n_{l, k}}+(-1)^{n_{j, k}}\right] \sqrt{P_{C_{k}}(0) P_{C_{k}}(1)}\right]
\end{aligned}
$$

and obtain

$$
\begin{aligned}
& \sum_{i=0}^{M-1} \gamma_{i, l} \gamma_{i, j}=\prod_{k=0}^{m-1}\left[(-1)^{n_{l, k}+n_{j, k}} P_{C_{k}}(0)+P_{C_{k}}(1)\right. \\
& \quad+\left[(-1)^{n_{l, k}}+(-1)^{n_{j, k}}\right] \sqrt{P_{C_{k}}(0) P_{C_{k}}(1)} \\
& \quad+(-1)^{n_{l, k}+n_{j, k}}\left((-1)^{n_{l, k}+n_{j, k}} P_{C_{k}}(0)+P_{C_{k}}(1)\right. \\
& \left.\left.\quad \quad-\left[(-1)^{n_{l, k}}+(-1)^{n_{j, k}}\right] \sqrt{P_{C_{k}}(0) P_{C_{k}}(1)}\right)\right] \\
& =\prod_{k=0}^{m-1}\left[\left((-1)^{n_{l, k}+n_{j, k}}+1\right) P_{C_{k}}(0)\right. \\
& \quad+\left(1+(-1)^{n_{l, k}+n_{j, k}}\right) P_{C_{k}}(1) \\
& \quad+\left[(-1)^{n_{l, k}}+(-1)^{n_{j, k}}-(-1)^{n_{j, k}}-(-1)^{n_{l, k}}\right] \\
& \left.\quad \cdot \sqrt{P_{C_{k}}(0) P_{C_{k}}(1)}\right] \\
& =\prod_{k=0}^{m-1}\left(1+(-1)^{n_{l, k}+n_{j, k}}\right),
\end{aligned}
$$

where the last step follows because $P_{C_{k}}(0)+P_{C_{k}}(1)=1$. The factors in (11) are either 2 or 0 , depending on whether $n_{l, k}=n_{j, k}$ or $n_{l, k} \neq n_{j, k}$ for the particular bit position $k$. Thus, the product will be zero unless all bits of $l$ and $j$ are equal, and

$$
\sum_{i=0}^{M-1} \gamma_{i, l} \gamma_{i, j}=\left\{\begin{array}{ll}
M, & j=l, \\
0, & j \neq l,
\end{array}, \quad l=0, \ldots, M-1 .\right.
$$

Applying (12) to the inner sum of (10) and dividing both sides by $M \sqrt{P_{j}}$, which by Sec. II is nonzero, completes the proof.

\section{NUMERICAL RESULTS}

Fig. 2 illustrates three alphabets: quaternary pulse amplitude modulation (4-PAM), eight-level star-shaped quaternary amplitude modulation (8-QAM), and an eight-level irregular constellation, which achieves the SL $-1.59 \mathrm{~dB}$ [9, Fig. 4 (a)]. Each alphabet is shown with two different input distributions, one uniform and one nonuniform, scaled to the the same average energy and translated to zero mean. The apparent threedimensional structure in Fig. 2 (c) is an illusion; the alphabet consists of eight points in the plane, just like Fig. 2 (b). An alphabet that admits this particular illusion can be described as a linear projection of a hypercube and achieves the SL at low SNR [9, Theorem 12] when used with a uniform distribution.

The BICM-MI of these constellations are is shown in Fig. 3. For 4-PAM and 8-QAM, probabilistic shaping improves the BICM-MI considerably over a wide range of SNRs, which is reasonable since the shaped constellations in Fig. 2 resemble 


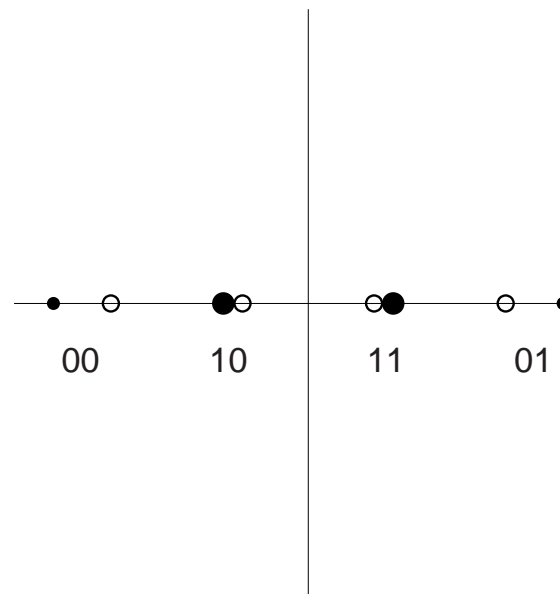

(a)

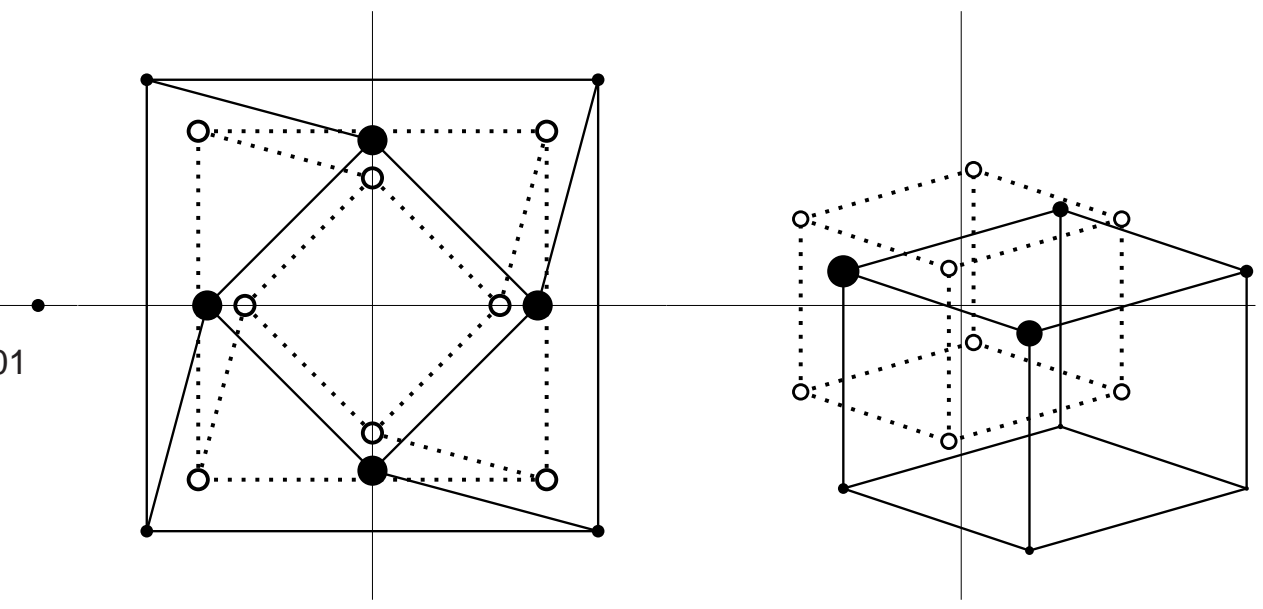

(b)

(c)

Fig. 2. Uniform $\left[\mathbb{X}, \mathbb{U}_{m}\right]$ (empty circles) and nonuniform (filled circles) constellations $[\mathbb{X}, \mathbb{P}]$ based on (a) 4-PAM, (b) star 8-QAM, and (c) an 8-level irregular alphabet. Lines connect symbols whose codewords have a Hamming distance of one. The symbol probabilities are proportional to the area of the corresponding circles. The constellations in (c) create an illusion of perspective, although they are purely two-dimensional just like those in (b).

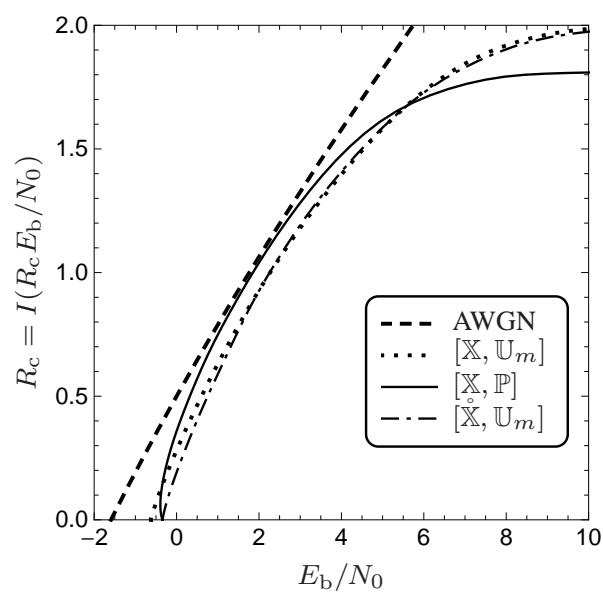

(a)

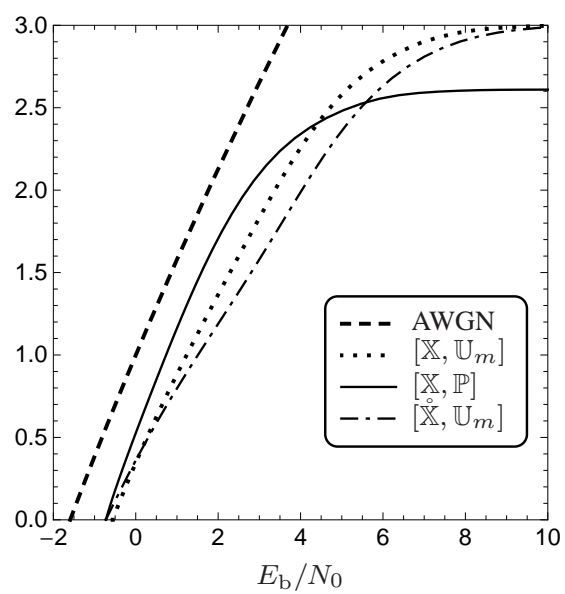

(b)

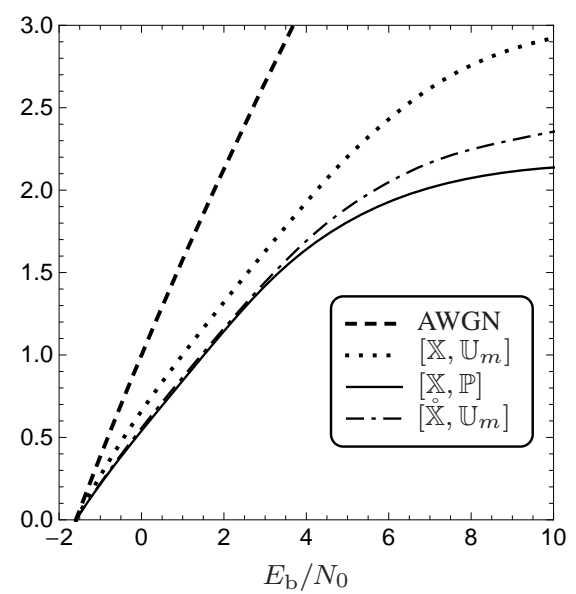

(c)

Fig. 3. The BICM-MI of the six constellations in Fig. 2 and the three transformed constellations in Fig. 4. The AWGN bound $(N / 2) \log 2(1+(2 / N) S N R)$ is included for reference, where the dimension $N=1$ for (a) and $N=2$ for (b) and (c). In all cases, the curves for $[\mathbb{X}, \mathbb{P}]$ and $\left[\dot{\mathbb{X}}, \mathbb{U}_{m}\right]$ meet at their lower endpoints, where $E_{\mathrm{b}} / N_{0}=\alpha^{-1}=-0.34$ (a), -0.73 (b), and $-1.59 \mathrm{~dB}$ (c).

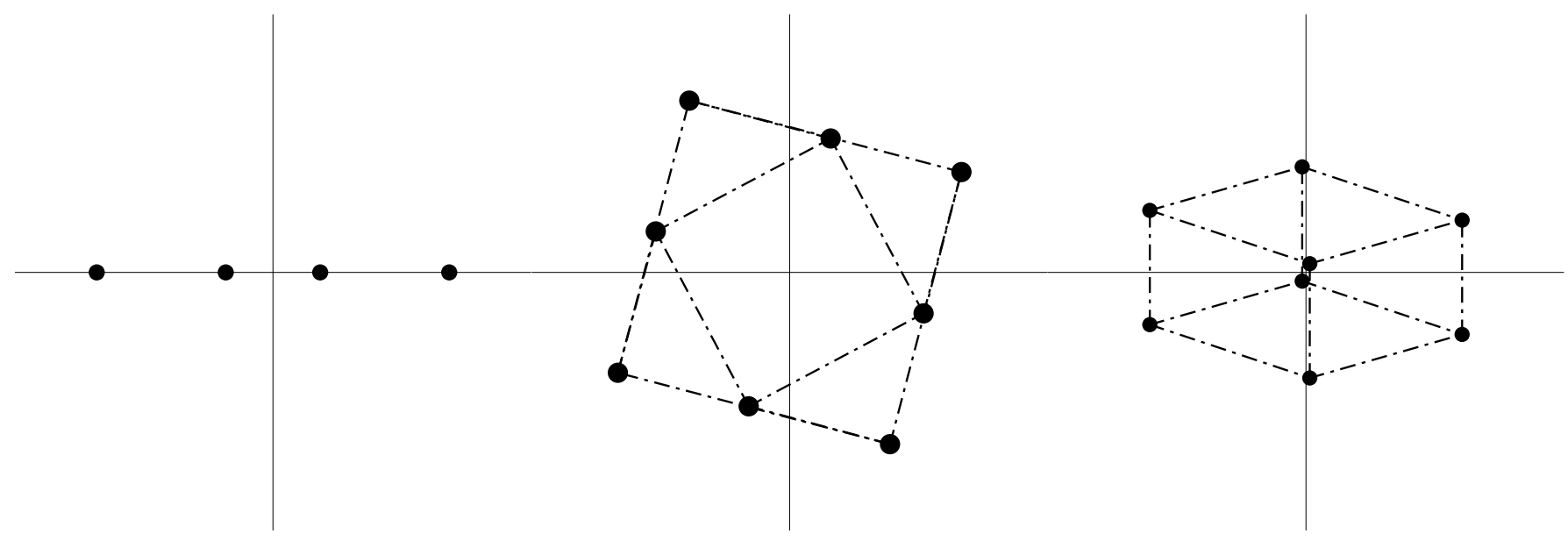

(a)

(b)

(c)

Fig. 4. The transforms $\left[\stackrel{\circ}{\mathbb{X}}, \mathbb{U}_{m}\right]$ of the three constellations $[\mathbb{X}, \mathbb{P}]$ in Fig. 2. 
a Gaussian distribution better. This is not the case for the third alphabet, whose BICM-MI is not improved by shaping, at least not with this particular distribution.

The transforms of the three nonuniform constellations are calculated according to (8) and evaluated with a uniform distribution. Graphically, the transformed constellations (shown in Fig. 4) look quite different from the original constellations in Fig. 2. The 4-PAM constellation with equal spacing and nonuniform probabilities is converted into a 4-PAM constellation with unequal spacing and uniform probabilities. The BICM-MIs of the transformed constellations, which are also shown in Fig. 3, are different from the original ones in general, with one exception: their lower endpoints coincide, indicating that their $\alpha$ parameters are the same. This equivalence between constellations and their transforms has been observed for every studied nonuniform constellation.

\section{CONCLUSIONS}

The numerical results in Sec. $\mathrm{V}$ provide evidence that the BICM-MI of two constellations related by the transform have the same low-SNR behavior, quantified by the parameter $\alpha$. Analytical evidence will be provided in a future publication. The significance of this relation lies in the abundance of existing analytical results for the first-order asymptotics of the BICM-MI with uniform distributions [5]-[9], [16] and the absence of similar results for nonuniform distributions.

\section{REFERENCES}

[1] E. Zehavi, "8-PSK trellis codes for a Rayleigh channel," IEEE Trans. Commun., vol. 40, no. 3, pp. 873-884, May 1992.

[2] A. Guillén i Fàbregas, A. Martinez, and G. Caire, "Bit-interleaved coded modulation," Foundations and Trends in Communications and Information Theory, vol. 5, no. 1-2, pp. 1-153, 2008.
[3] A. Martinez, A. Guillén i Fàbregas, and G. Caire, "Bit-interleaved coded modulation revisited: A mismatched decoding perspective," IEEE Trans. Inf. Theory, vol. 55, no. 6, pp. 2756-2765, June 2009.

[4] G. Caire, G. Taricco, and E. Biglieri, "Bit-interleaved coded modulation," IEEE Trans. Inf. Theory, vol. 44, no. 3, pp. 927-946, May 1998.

[5] A. Martinez, A. Guillén i Fàbregas, and G. Caire, "Bit-interleaved coded modulation in the wideband regime," IEEE Trans. Inf. Theory, vol. 54, no. 12 , pp. 5447-5455, Dec. 2008.

[6] A. Alvarado, E. Agrell, A. Guillén i Fàbregas, and A. Martinez, "Corrections to 'Bit-interleaved coded modulation in the wideband regime'," IEEE Trans. Inf. Theory, vol. 56, no. 12, p. 6513, Dec. 2010.

[7] C. Stierstorfer and R. F. H. Fischer, "Mappings for BICM in UWB scenarios," in International ITG Conference on Source and Channel Coding (SCC), Ulm, Germany, Jan. 2008.

[8] - , Asymptotically optimal mappings for BICM with $M$-PAM and $M^{2}$-QAM," IET Electronics Letters, vol. 45, no. 3, pp. 173-174, Jan. 2009.

[9] E. Agrell and A. Alvarado, "Optimal alphabets and binary labelings for BICM at low SNR," IEEE Trans. Inf. Theory, vol. 57, no. 10, pp. 6650-6672, Oct. 2011.

[10] S. Y. Le Goff, B. S. Sharif, and S. A. Jimaa, "A new bit-interleaved coded modulation scheme using shaping coding," in IEEE Global Telecommunications Conference (GLOBECOM), Dallas, TX, Nov.-Dec. 2004.

[11] D. Raphaeli and A. Gurevitz, "Constellation shaping for pragmatic turbo-coded modulation with high spectral efficiency," IEEE Trans. Commun., vol. 52, no. 3, pp. 341-345, Mar. 2004.

[12] S. Y. Le Goff, B. S. Sharif, and S. A. Jimaa, "Bit-interleaved turbocoded modulation using shaping coding," IEEE Commun. Lett., vol. 9, no. 3, pp. 246-248, Mar. 2005.

[13] S. Y. Le Goff, B. K. Khoo, C. C. Tsimenidis, and B. S. Sharif, "Constellation shaping for bandwidth-efficient turbo-coded modulation with iterative receiver,' IEEE Trans. Wireless Commun., vol. 6, no. 6, pp. 2223-2233, June 2007.

[14] A. Guillén i Fàbregas and A. Martinez, "Bit-interleaved coded modulation with shaping," in IEEE Information Theory Workshop (ITW), Dublin, Ireland, Aug.-Sep. 2010.

[15] T. Nguyen and L. Lampe, "Bit-interleaved coded modulation with mismatched decoding metrics," IEEE Trans. Commun., vol. 59, no. 2, pp. 437-447, Feb. 2011.

[16] C. Stierstorfer and R. F. H. Fischer, "(Gray) Mappings for bit-interleaved coded modulation," in IEEE Vehicular Technology Conference (VTCSpring), Dublin, Ireland, Apr. 2007. 